\title{
Effects of rapidly decaying plasmas on Langmuir probe measurements
}

\author{
Guowen Ding, John E. Scharer, ${ }^{\text {a) }}$ and Kurt L. Kelly \\ Department of Electrical and Computer Engineering, University of Wisconsin-Madison, \\ Wisconsin 53706-1687
}

(Received 9 July 1997; accepted for publication 23 April 1998)

\begin{abstract}
Sheath motion, displacement current, and probe edge effects on temporal Langmuir probe (LP) ion saturation current measurements are investigated for a pulsed laser produced plasma during the period $100 \mathrm{~ns} \rightarrow 1000 \mathrm{~ns}$ after the turn on of the laser pulse. The plasma has a large volume (hundreds of $\mathrm{cm}^{3}$ ) and a high initial plasma density $\left(n_{e}>10^{13} \mathrm{~cm}^{-3}\right)$. The sheath motion and edge effects are found to be very important, but the displacement current is found to be very small. We present both a quantitative correction for the effects of rapidly decaying plasmas on LP ion saturation current measurements and a validity condition for this method. The results are compared with the densities predicted from electron saturation currents, and the former are $\leqslant 30 \%$ lower than the latter. The corrected probe measurements are utilized to determine the plasma recombination coefficient. It is found to be in good agreement with the results obtained by Stalder and Eckstrom [J. Appl. Phys. 72, 3917 (1992)] who utilized a microwave method. (C) 1998 American Institute of Physics. [S0021-8979(98)00815-9]
\end{abstract}

\section{INTRODUCTION}

Most Langmuir probe (LP) measurements are carried out in slowly varying or steady-state plasmas. Recently, timeresolved probe measurements of radio frequency plasmas have been reported ${ }^{1,2}$ where displacement currents are important. However, little attention has been paid to the issue of how to measure a rapidly decaying plasma by a LP where the sheath motion effect is more important than displacement current.

Our pulsed, vacuum-ultraviolet laser can create a $100 \mathrm{~cm}^{3}$ volume plasma in an organic gas with high plasma density $\left(n_{e}>10^{13} \mathrm{~cm}^{-3}\right)$ on a $10 \mathrm{~ns}$ time scale. ${ }^{3,4}$ If the plasma ions are multiatom molecular ions, then the plasma recombination coefficient is very large, so the plasma density decays rapidly, especially for high plasma densities. For example, for a tetrakis(dimethyl-amino)ethylene (TMAE) molecular ion with 38 atoms, a plasma density of $10^{13} \mathrm{~cm}^{-3}$ decays to $10^{11} \mathrm{~cm}^{-3}$ on a $\mu$ s time scale. The rapidly decaying plasma has an important influence on LP ion saturation current measurements, which is absent in a steady-state plasma.

In our experiment, a rapidly decaying plasma is created by $193 \mathrm{~nm}$ excimer laser ionization of the organic molecule TMAE. Utilizing this laser and gas combination, Zhang and Scharer $^{3}$ have obtained a laser produced TMAE plasma as well as microwave reflections from it, and Shen et al. ${ }^{4}$ have demonstrated that the laser can produce a large TMAE plasma sheet and discussed the resulting detailed spatial and temporal plasma profiles. These LP measurements are qualitatively correct, but have neglected the rapidly decaying plasma influence on LP measurements, which is considered in this letter.

${ }^{a)}$ Electronic mail: scharer@engr.wisc.edu
In this letter, fast decaying plasma effects are studied for the $100 \mathrm{~ns} \rightarrow 1000 \mathrm{~ns}$ period after the turn on of the laser pulse which has a duration of $20 \mathrm{~ns}$. The probe sheath motion as well as probe edge effects are found to be very important in LP ion saturation current measurements effects, but the displacement current effect is found to be small. Utilizing sheath motion theory, ${ }^{5-7}$ we present a quantitative correction for both sheath motion and probe edge effects. The validity condition for this method is presented as well.

In order to compare our LP measurements with measurements by a microwave method obtained by Stalder and Eckstrom, ${ }^{8}$ the LP plasma density obtained is used to calculate the TMAE plasma recombination coefficient and it is compared with their results. We also compare the electron densities obtained from the corrected ion saturation currents with those obtained from electron saturation currents and the former are $\leqslant 30 \%$ lower than the latter. Comparing these two methods, the electron saturation current method requires experimental conditions which are repeatable for hundreds of shots to obtain an accurate $I-V$ characteristics, but no sheath effects are considered, because the small bias voltages (plasma potentials $\leqslant 1.1 \mathrm{~V}$ ) give rise to very small sheath effects. On the other hand, the ion saturation method is much simpler with only one temporal measurement, but sheath effects must be considered.

\section{EXPERIMENTAL APPARATUS}

A schematic experimental arrangement is illustrated in Fig. 1. A laboratory plasma is created in a $50 \mathrm{~cm}$ long by $15.2 \mathrm{~cm}$ diam cylindrical glass chamber, which is pumped to a base pressure of $1 \times 10^{-6}$ Torr by a diffusion pump. A Suprasil window which is transparent down to $180 \mathrm{~nm}$ wavelengths is mounted on the laser entrance end of the vacuum chamber. On the opposite side of the chamber, a rotatable 


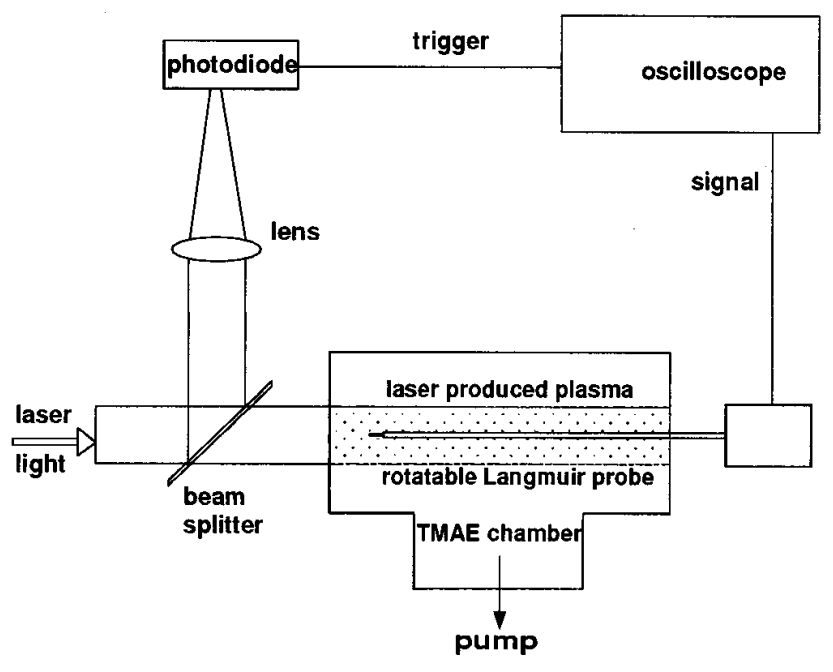

FIG. 1. Schematic of the experimental arrangement.

Langmuir probe and a flow feed to introduce the TMAE vapor are inserted through feedthroughs to the vacuum chamber.

A Lumonics PM842 excimer laser runs in an ArF mode and produces $193 \mathrm{~nm}$ wavelength radiation. The half-width of the laser pulse is $20 \mathrm{~ns}$. The shape of laser output is a $3 \mathrm{~cm} \times 1.3 \mathrm{~cm}$ rectangle. The laser intensity in the central $2 \mathrm{~cm} \times 0.7 \mathrm{~cm}$ of the laser beam is quite uniform, $\Delta I / I$ $\leqslant 10 \%$ as measured by an apertured photodiode. The probe signals are measured in this area.

The plasma parameters are primarily detected by a single Langmuir probe. The Langmuir probe for ion saturation current measurement used in this experiment consists of a double-shielded $50 \Omega$ coaxial cable spot welded to a $2.4 \mathrm{~mm}$ diam tantalum tip. The probe shaft extending out of the chamber is directly connected to a shielded probe circuit which is shown in Fig. 2. A $20 \mathrm{~cm}$ long cable connects this circuit to a fast Tektronix digital storage oscilloscope (Model TDS $350,1 \mathrm{GHz}$ ). The probe surface can be rotated so that it is parallel to the laser beam. The probe current is measured by the voltage across a $50 \Omega$ resistor, matching the cable impedance. The response time for the probe circuit is $7 \mathrm{~ns}$, which is sufficiently rapid for our LP measurements.

In order to check our results obtained from corrected ion saturation current measurements. we used another small single-sided probe to detect electron saturation current due to



FIG. 2. Circuit for measuring the probe current. the high electron current. The probe is the cross section of the end of a $1 \mathrm{~mm}$ diameter tungsten wire, the sides of which are insulated by a close fitting ceramic tube with the last 0.05 $\mathrm{mm}$ of the sides exposed. The wire is connected to a doubleshielded $50 \Omega$ coaxial cable. The collection current area of the probe is 11.5 times smaller than that of the $2.4 \mathrm{~mm}$ diameter disk double-sided probe, so that the load voltage on the resistor $\left(R_{s}=50 \Omega\right)$ is significantly reduced. We vary the bias voltage on the circuit from -3 to $+4 \mathrm{~V}$ in increments of $0.05 \mathrm{~V}$, and then they are corrected for the load voltage on the resistor to obtain the net bias voltage. During the period from 200 to $900 \mathrm{~ns}$. the floating potentials vary from -0.4 $\rightarrow-0.5 \mathrm{~V}$, and the corresponding plasma potentials are 1.1 $\rightarrow 0.9 \mathrm{~V}$. The plasma potentials are determined from the maxima of the current derivative with respect to voltage, then the electron saturation currents can also be determined. No sheath effects have been considered for this case since the small bias voltage (plasma potentials $\leqslant 1.1 \mathrm{~V}$ ) has a negligible sheath effect.

\section{THEORY}

In steady state, the ion saturation current density of a Langmuir probe is given by the Bohm current density

$$
J_{B}=0.6 \text { nev }_{B},
$$

where $n$ is plasma density, $v_{B}$ is the Bohm velocity $\sqrt{T_{e} / M_{i}}$, and $T_{e}$ and $M_{i}$ are the electron temperature and the ion mass, respectively.

However, when the sheath motion is comparable to $v_{B}$, a correction for the LP measurement is needed. According to sheath motion theory, ${ }^{6}$ the total current density is

$$
J=0.6 n e\left(v_{B}+\frac{d s}{d t}\right),
$$

where $s$ is the sheath width and $d s / d t$ is the velocity of the sheath edge.

To solve Eq. (2), the sheath velocity must be determined, and it can be calculated from the Child-Langmuir law

$$
J=\frac{4}{9} \sqrt{\frac{2 e}{M_{i}}} \frac{\epsilon_{0} V^{3 / 2}}{s^{2}},
$$

where $V$ is the sheath voltage. When the bias voltage is high enough, it is much larger than the plasma potential, so that the sheath voltage is close to the bias voltage.

Combining Eqs. (1), (2), and (3), we obtain

$$
J_{B}=\frac{J}{1+\frac{2}{3}\left(\frac{\epsilon_{0}}{T_{e}}\right)^{1 / 2}\left(2 e M_{i}\right)^{1 / 4} V^{3 / 4} \frac{d\left(J^{-1 / 2}\right)}{d t}} .
$$

The physical meaning of Eq. (4) is that the sheath motion contributes to the LP current in addition to that from the Bohm current. If the sheath motion effect is small or $d J / d t$ is small, Eq. (4) reduces to $J_{B}=J$, which is the result for a Langmuir probe under steady-state conditions. This correction is related not only to the time rate of change of plasma density, but also to the sheath voltage $V$ and the electron temperature $T_{e}$. 
Probe edge effects can increase the effective collecting area for the probe current. For a double-sided planar probe disk, the measured probe current $I_{m}$ can be expressed as

$$
I_{m}=J\left(2 \pi r^{2}+2 \pi r \pi s\right),
$$

where $r$ is the probe radius. The sheath width $s$ is also calculated from the Child-Langmuir law, so $J$ can be determined by

$$
\frac{I_{m}}{2 \pi r^{2}}=J+\frac{2 \pi}{3 r} \epsilon_{0}^{1 / 2}\left(\frac{2 e}{M_{i}}\right)^{1 / 4} V^{3 / 4} J^{1 / 2} .
$$

Equation (6) shows that the probe edge effect increases as $J$ decreases, so the probe edge effect increases with time for a rapidly decaying plasma.

The above analysis requires quasistatic conditions so that the Child-Langmuir law is valid. This assumption means that the plasma parameters are almost constant within a sheath response time, which is represented by the time scale of the inverse ion plasma frequency $\omega_{p i} .{ }^{9}$ We then determine whether or not the change in the electron density $\Delta n_{e}$ within a time scale of $1 / \omega_{p i}$ is much smaller than the electron density $n_{e}\left(\left|\Delta n_{e}\right| \ll n_{e}\right)$.

In our experiment, after the laser pulse is terminated, there are primarily two processes, a two-body recombination process and a collisional ionization process between electrons and excited molecules. These processes are described as

$$
\frac{d n_{e}}{d t}=-\alpha n_{e}^{2}+\nu n_{e} n^{*},
$$

where $\alpha$ is the two-body recombination coefficient, $n^{*}$ is the excited molecular density, and $\nu$ is the rate of electronexcited molecular ionization.

We substitute $d n_{e}=\Delta n_{e}$ and $d t=1 / \omega_{p i}=\sqrt{\epsilon_{0} M_{i} / e^{2} n_{e}}$ into Eq. (7), so the validity condition $\left|\Delta n_{e}\right| \ll n_{e}$ becomes

$$
n_{e} \ll \frac{e^{2}}{\left(\alpha-\nu n^{*} / n_{e}\right)^{2} \epsilon_{0} M_{i}} .
$$

This condition can be simplified to

$$
n_{e} \ll \frac{e^{2}}{\alpha^{2} \epsilon_{0} M_{i}},
$$

because once this equation is satisfied, Eq. (8) is surely satisfied.

For TMAE, $\alpha$ has been previously measured to be $9 \times 10^{-6} \mathrm{~cm}^{3} / \mathrm{s}$, ${ }^{8}$ so Eq. (9) requires $n_{e} \ll 1.1 \times 10^{14} \mathrm{~cm}^{-3}$ for our case. During the period $100 \mathrm{~ns}<t<1000 \mathrm{~ns}$, our plasma density is in the range of $3 \times 10^{12}-10^{11} \mathrm{~cm}^{-3}$, which is much smaller than $10^{14} \mathrm{~cm}^{-3}$, so the quasistatic assumption is valid for our experimental conditions.

The quasistatic assumption implies that the displacement current is small. The displacement current can be roughly estimated if we assume a uniform electric field in the sheath

$$
J_{d}=\epsilon_{0} \frac{d(V / s)}{d t} .
$$

The quasistatic assumption implies that the plasma density is almost constant within $d t=1 / \omega_{p i}$, so the sheath width $s$ is

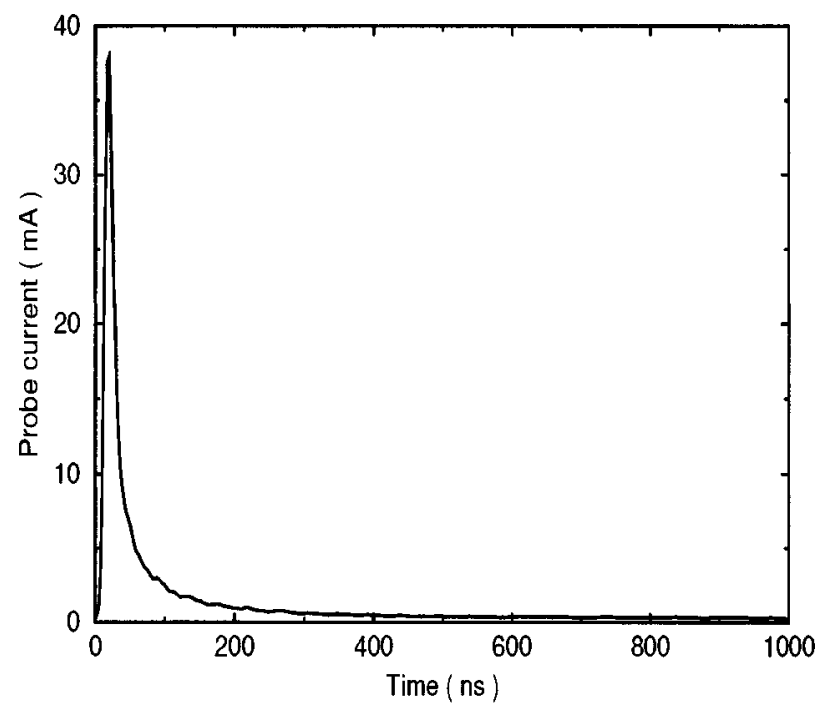

FIG. 3. Langmuir probe measurement for TMAE plasma. (The bias voltage is $-18 \mathrm{~V}$.)

almost constant according to the Child-Langmuir law. Thus, a constant bias voltage $V$ and sheath width $s$ in Eq. (10) result in negligible displacement currents.

In summary, Eqs. (4) and (6) can be used for corrections of LP measurement in measuring a rapidly decaying plasma, and the displacement current can be neglected, as long as Eq. (9) is satisfied.

\section{RESULTS AND DISCUSSION}

In order to check our theory, we investigate a rapidly decaying plasma. The plasma is created under $75 \mathrm{mTorr}$ TMAE pressure conditions and a $3 \mathrm{~mJ} / \mathrm{cm}^{2}$ laser pulse flux density. The Langmuir probe signal begins with a very high peak, then decays rapidly and continues with a more gradually decaying signal, which is shown in Fig. 3. This peak is related to the process of the laser produced plasma. At this stage, the probe sheath is forming, so the transient ion current, displacement current, as well as laser effects on the probe are expected to influence the probe current. After the laser is turned off, the laser effects are eliminated. Then after several $\omega_{p i}^{-1}$, or $t>100 \mathrm{~ns}$, the transient ion current becomes very small. In the meantime, the probe sheath is formed, and the displacement current is small as well due to the quasistatic condition discussed in the theory section.

Thus, for $t>100 \mathrm{~ns}$, the number of physical processes influencing the probe current are reduced. The probe current density primarily consists of Bohm and sheath motion current densities, and the probe collecting area is influenced by the sheath around the probe edges. Considering these effects, we present a method in the theory section to calculate the plasma density from the probe current.

The experimentally measured and corrected probe currents are shown in Fig. 4(a), and the correction factors are shown in Fig. 4(b). The probe current correction arises from both sheath motion and probe edge effects. The corrected probe current in Fig. 4(a) is the product of the Bohm current density and the probe area. The sheath motion effect de- 

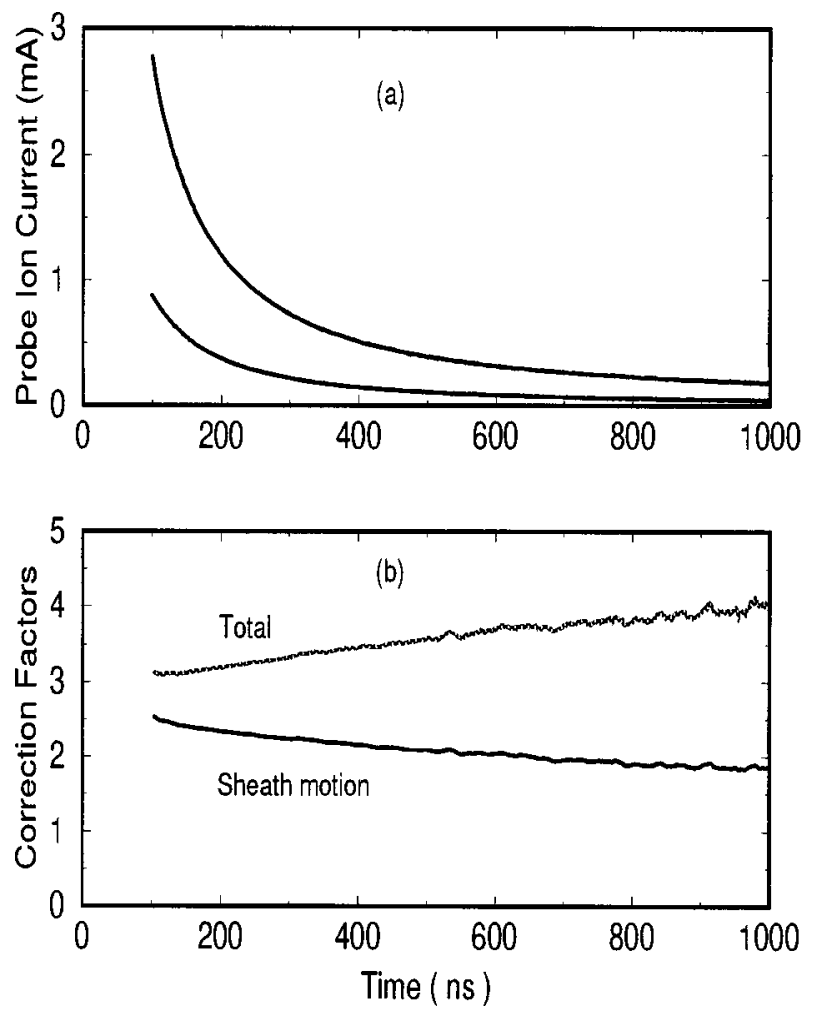

FIG. 4. (a) Probe ion saturation current and its correction for both sheath motion and probe edge effects. (b) Probe current correction factors (the bias voltage is $-67 \mathrm{~V})$.

creases with time as shown in Fig. 4(b), since sheath motion is more rapid at earlier times. On the other hand, probe edge effects increase with time, since the sheath width is smaller for the higher plasma densities at earlier times. Thus, the total correction for the two effects slightly increases with time as shown in Fig. 4(b).

The probe $I-V$ characteristics from the $2.4 \mathrm{~mm}$ diam double-sided probe, before and after the corrections are shown in Fig. 5. One readily observes that the corrected ion currents are much less dependent on the bias voltage. This agrees with the principle that the ideal ion saturation current is independent of the bias voltage.

The electron temperature is $0.3 \mathrm{eV}$, which is determined from the slope of the logarithmic $I-V$ characteristics from a $1 \mathrm{~mm}$ diam single-sided probe. This result agrees with the laser photon energy of $6.4 \mathrm{eV}$ and a TMAE vertical ionization potential of $6.1 \mathrm{eV} .^{10}$

The plasma potentials are determined from the maxima of $d I / d V$, from which the electron saturation currents can be determined. The $I-V$ characteristic curves and $d I / d V$ are shown in Figs. 6(a) and 6(b). The curves are averages of eight laser shots with a standard deviation of $5 \%$. The plasma densities determined by both electron saturation currents and ion saturation currents corrected by our theory are shown in Fig. 7. The latter is $30 \%$ smaller than the former. This implies that our theoretical model for correction of ion saturation current is very good, where the correction factors are more than three as shown in Fig. 4(b).

Another way to check our theory for evaluation of ion saturation measurements is to calculate the TMAE plasma

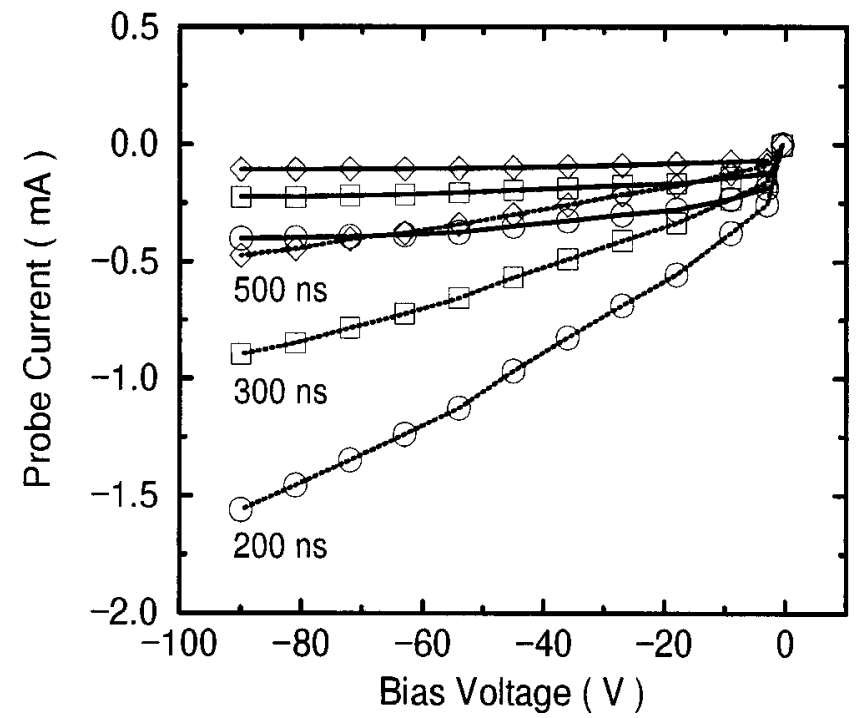

FIG. 5. $I-V$ characteristics from a $2.4 \mathrm{~mm}$ diameter double-sided probe, before (dotted line) and after corrections (solid line) for both sheath motion and probe edge effects (for various delay times from the initial laser pulse).

recombination coefficient, and compare it with that of Stalder and Eckstrom ${ }^{8}$ who used a microwave method. Although they introduced a TMAE seed gas under atmospheric-pressure helium conditions, the three-body recombination effect is small, where the total recombination coefficient $\quad \alpha_{\text {total }}=\alpha_{\text {two-body }}+\beta_{\text {three-body }} \times n_{\text {atom }}$. Pitaevskii's formula ${ }^{11}$ predicts that the three-body recombination coefficient $\beta$ for $e+\mathrm{TMAE}^{+}+\mathrm{He}$ is close to that for $e$ $+\mathrm{He}_{2}^{+}+\mathrm{He}$, which is known to be $\beta=2 \times 10^{-27} \mathrm{~cm}^{6} / \mathrm{s}$ at $300 \mathrm{~K} .{ }^{12}$ For atmospheric pressure $\left(n_{\text {atom }} \approx 2.7\right.$ $\left.\times 10^{19} \mathrm{~cm}^{-3}\right)$, and $\beta \times n_{\text {atom }}$ is $5 \times 10^{-8} \mathrm{~cm}^{3} / \mathrm{s}$. Since the TMAE plasma two-body recombination coefficient is so large $\alpha \approx 9 \times 10^{-6} \mathrm{~cm}^{3} / \mathrm{s}$, the three-body recombination effect is small. Although the electron temperatures for these two cases are different, room temperature and $0.3 \mathrm{eV}$, respectively, both the theory and experiments show that the
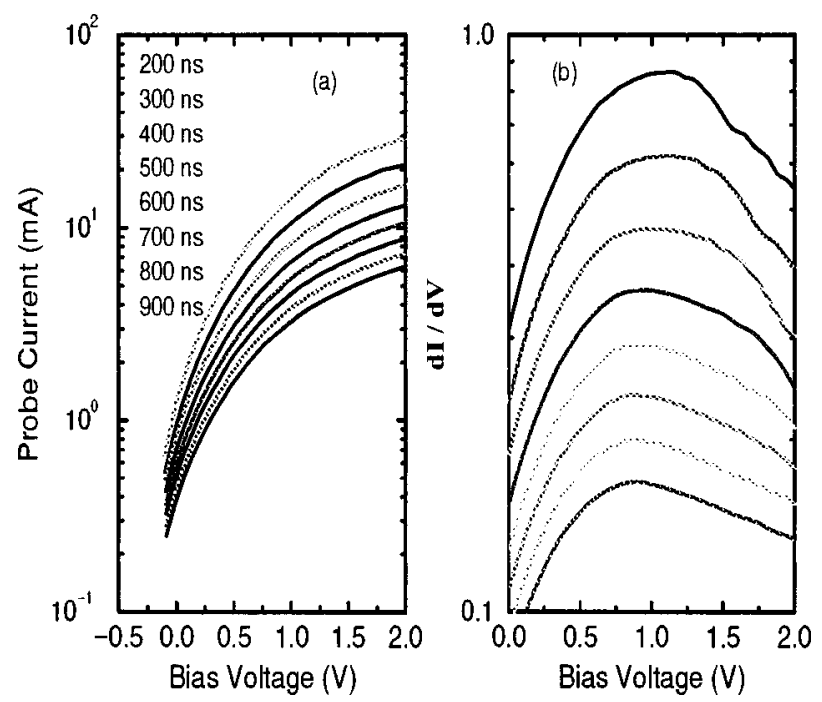

FIG. 6. (a) $I-V$ characteristics for a $1 \mathrm{~mm}$ diameter single-sided probe, and (b) $d I / d V$. 


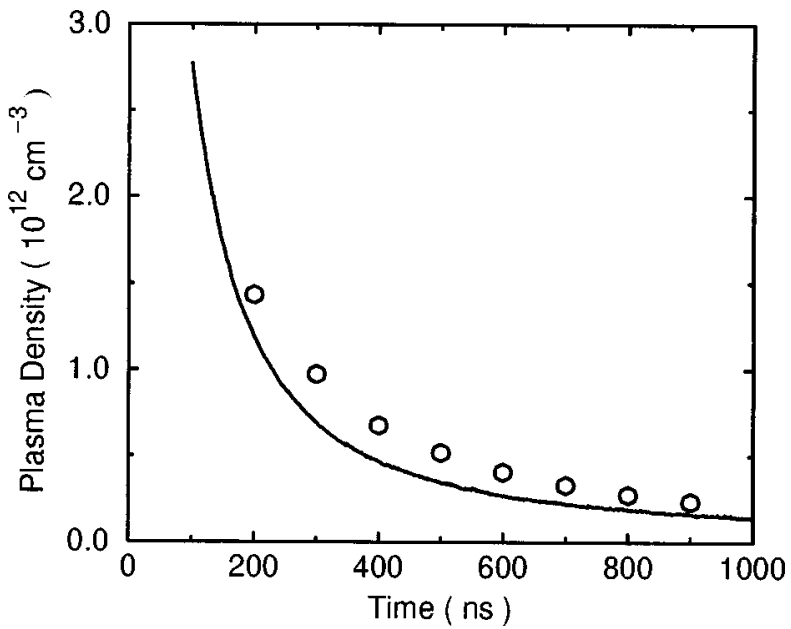

FIG. 7. Plasma density vs time, obtained from the ion saturation currents corrected by the theory (solid line), and from the electron saturation currents (circles).

plasma recombination coefficient for a complex molecular ion is almost independent of electron temperature, which has been shown to be the case for $\mathrm{H}_{3} \mathrm{O}^{+}\left(\mathrm{H}_{2} \mathrm{O}\right)_{2}$ and $\mathrm{NH}_{4}^{+}\left(\mathrm{NH}_{3}\right)_{2}$ plasmas. ${ }^{13,14}$ Thus, the TMAE plasma recombination coefficient $\alpha$ should not severely be affected by the different experimental conditions.

In our experiment, the recombination coefficient $\alpha$ can be accurately obtained for $t>400 \mathrm{~ns}$. This is because in this stage the electron-excited molecular collisional ionization is negligible, so two-body recombination is dominant. The differential equation describing this process can be expressed as

$$
\frac{d n_{e}}{d t}=-\alpha n_{e}^{2}
$$

It has the well-known solution

$$
\frac{1}{n_{e}}=\frac{1}{n_{e}\left(t_{0}\right)}+\alpha t .
$$

A plot of $1 / n_{e}$ versus time is shown in Fig. 8, and its slope yields $\alpha$. Utilizing this method, our result for the recombination coefficient is $\alpha=8.0( \pm 1.5) \times 10^{-6} \mathrm{~cm}^{3} / \mathrm{s}$. This is in good agreement with the microwave measurement ${ }^{8}$ which obtained $\alpha=9.0( \pm 1.1) \times 10^{-6} \mathrm{~cm}^{3} / \mathrm{s}$ for a TMAE plasma. Since the plasma density is primarily determined by the recombination coefficient $\alpha$, time and initial density, an accurate recombination coefficient implies accurate plasma density measurements. This provides confidence in the accuracy of our method and the corrections utilized for obtaining density from ion saturation measurements under rapidly varying plasma density conditions.

\section{SUMMARY}

For a pulsed laser produced plasma, the sheath motion effect is found to be important in the interpretation of Langmuir probe measurements. For a small diameter probe, the probe edge effect must be considered as well. The displace-

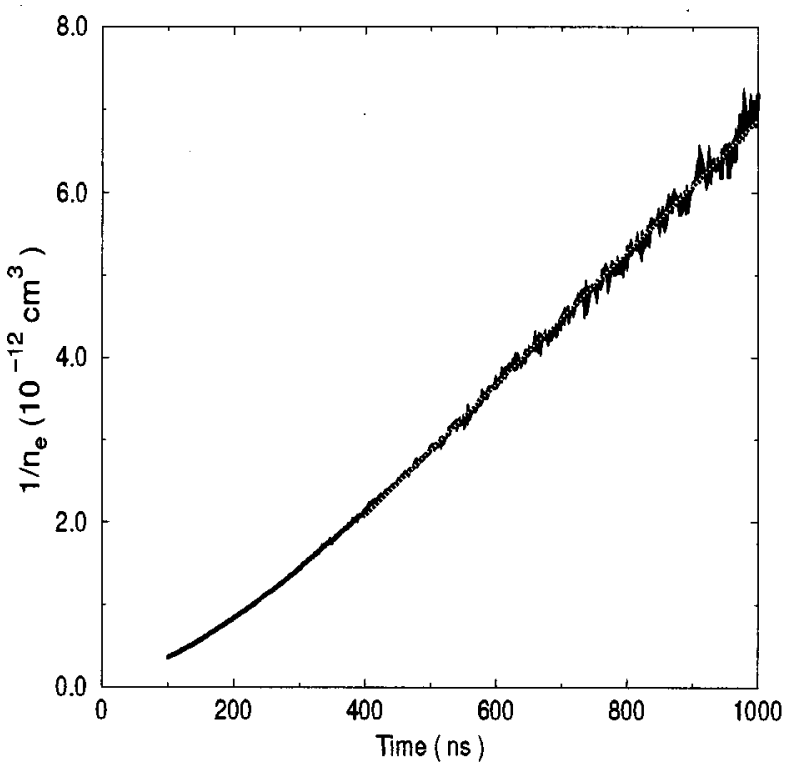

FIG. 8. Relation between $1 / n_{e}$ and $t$.

ment current in our case is found to be very small and is neglected. The sheath motion effect decreases with time, and the probe edge effect increases with time. We present both a quantitative correction for fast decaying plasma effects on LP measurements and a validity condition for this method. The results obtained by this method are in agreement with those obtained by the electron saturation current method, and agrees with the plasma recombination coefficient measured by a microwave method.

\section{ACKNOWLEDGMENTS}

The authors wish to thank Drs. M. Bettenhausen and N. Lam for valuable discussions. This work is primarily supported by Air Force Office of Scientific Research Grants (Grants F49620-94-1-0054 and F49620-97-1-0262) in cooperation with the Defense Department Research and Engineering Air Plasma Ramparts Multi University Research Initiative program. It is also supported in part by the University of Wisconsin.

${ }^{1}$ R. B. Tukot, Jr. and D. N. Ruzic, J. Appl. Phys. 73, 2173 (1993).

${ }^{2}$ M. Klick, J. Appl. Phys. 79, 3445 (1996).

${ }^{3}$ Y. S. Zhang and J. E. Scharer, J. Appl. Phys. 73, 4779 (1993).

${ }^{4}$ W. Shen, J. E. Scharer, B. G. Porter, N. T. Lam, and K. Kelly, J. Appl. Phys. 78, 6974 (1995).

${ }^{5}$ J. K. Chester, Journal of Science \& Technology 37, 2 (1970).

${ }^{6}$ F. F. Chen, Phys. Fluids 25, 2385 (1982).

${ }^{7}$ M. A. Lieberman, J. Appl. Phys. 66, 2926 (1989).

${ }^{8}$ K. R. Stalder and D. J. Eckstrom, J. Appl. Phys. 72, 3917 (1992).

${ }^{9}$ F. F. Chen, Plasma Diagnostic Techniques, edited by R. H. Huddlestone and S. L. Leonard (Academic, New York, 1965), Chap. 4.

${ }^{10}$ Y. Nakato, M. Ozaki, A. Egawa, and H. Tsubomura, Chem. Phys. Lett. 9, 615 (1971).

${ }^{11}$ L. P. Pitaevskii, Sov. Phys. JETP 15, 919 (1962).

${ }^{12}$ J. Berlande, M. Cheret, R. Deloche, A. Gonfalone, and C. Manus, Phys. Rev. A 1, 887 (1970).

${ }^{13}$ M. A. Biondi, Principles of Laser Plasmas, edited by G. Bekefi (Wiley, New York, 1976), Chap. 4, pp. 127-146.

${ }^{14}$ C. Huang, M. Whitaker, M. A. Biondi, and R. Johnsen, Phys. Rev. A 18, 64 (1978). 